\title{
Reabilitação oral de paciente com síndrome da combinação: relato de caso
}

\section{Patient oral rehabilitation with combination syndrome: case report}

\author{
Bruna Fernandes Rodrigues de Oliveira* \\ Isadora Sousa Arruda** \\ José James de Negreiros Pereira*** \\ Manassés Tercio Vieira Grangeiro ${ }^{* * *}$ \\ Poliana Lima Bastos ${ }^{* * * *}$ \\ Viviane Maria Gonçalves de Figueiredo ${ }^{* * * * *}$
}

\section{Resumo}

Objetivo: o tratamento de pacientes desdentados com reabsorção alveolar severa é uma temática desafiadora na reabilitação protética. Identificada por Kelly em 1972, a síndrome da combinação é uma manifestação intraoral encontrada em pacientes totalmente desdentados na arcada superior, em oposição a uma prótese mandibular de extensão distal bilateral. Relato de caso: este estudo revisa e discute, por meio de um caso clínico, as características clínicas presentes em um paciente com manifestações da síndrome da combinação. Além disso, apresenta uma proposta de tratamento reabilitador, restabelecendo a função fonética e a estética do paciente. Considerações finais: diagnosticar a síndrome e estabelecer um plano de tratamento adequado às necessidades do paciente, criando condições clínicas para o restabelecimento da saúde, são imprescindíveis para obter resultado e, assim, impedir o avanço do quadro clínico.

Palavras-chave: Diagnóstico. Síndrome de Kelly. Síndrome da combinação.

\section{Introdução}

É comum a reabilitação de pacientes desdentados superiores e parcialmente desdentados inferiores em clínicas odontológicas. Em geral, pacientes com a presença apenas de dentes anteriores inferiores e reabsorções específicas são muito comuns. ${ }^{1,2}$ Alterações nos tecidos dos palatos duro e mole na maxila foram relatadas nos pacientes com uso de próteses totais em oposição a remanescentes dentários inferiores anteriores e com extensão distal bilateral em prótese parcial removível..$^{3-5}$ As alterações baseiam-se em perda óssea na região anterior da crista maxilar, hipertrofia das tuberosidades, hiperplasia papilar no palato duro, extrusão dos dentes anteriores inferiores e reabsorção óssea dos rebordos alveolares distais bilaterais da mandíbula, ${ }^{3}$ referindo-se à síndrome da combinação. Sendo assim, acarretam a evolução ao longo do tempo de condições patológicas do sistema estomatognático. ${ }^{1}$ Posteriormente são descritas seis alterações adicionais associadas com a síndrome da cominação: diminuição da dimensão vertical de oclusão; discrepância do plano oclusal; reposicionamento espacial anterior da mandíbula; má adaptação das próteses; epulis fissuratum; e alterações periodontais. ${ }^{4}$

Quando o paciente tem apenas os dentes inferiores anteriores e é usuário de prótese total superior, a sobrecarga oclusal sobre a maxila anterior é potencializada, já que o paciente tende a exceder de maior força, justificando a maior reabsorção, e ainda ser capaz de desenvolver epullis fissuratum. ${ }^{3}$ Foi observado que pacientes com síndrome da combinação possuem dificuldade com o uso contínuo

Graduanda de Odontologia, Universidade Federal do Ceará, Sobral, CE, Brasil.

Graduanda de Odontologia, Universidade Federal do Ceará, Sobral, CE, Brasil.

Graduando de Odontologia, Universidade Federal do Ceará, Sobral, CE, Brasil.

Mestrando de Prótese Dentária, Unesp, São José dos Campos, SP, Brasil.

Doutora em Clínica Odontológica, professora da Faculdade de Odontologia, Universidade Federal do Ceará, Sobral, CE, Brasil.

Doutora em Prótese Dentária, professora do Curso de Odontologia da Unipê, João Pessoa, PB, Brasil. 
da prótese, necessitando de correção cirúrgica para melhor funcionalidade. ${ }^{6-8}$

A longo prazo, esse aspecto frequentemente inclui instabilidade nas próteses que, se não atenuada, leva a uma reabsorção alveolar posterior da mandíbula, mesmo que em evolução lenta e progressiva, passando despercebida pelo paciente e pelo cirurgião-dentista. ${ }^{9}$ Conhecer essas alterações é de extrema relevância para o cirurgião-dentista diagnosticar e efetuar um plano de tratamento eficaz, a fim de uma melhor reabilitação do paciente. ${ }^{3,10,11}$ Embora essas mudanças sejam reconhecidas, e haja diversos tratamentos, ${ }^{3,12,13}$ há poucos relatos documentados na literatura. ${ }^{1,9,12}$

As perspectivas de reabilitação em pacientes desdentados totais na maxila e bilateralmente posterior na mandíbula são: confecção de prótese total na maxila e prótese parcial removível na mandíbula, instalação de implantes osseointegrados para confecção de próteses com abutments tanto superior como inferior e próteses parciais fixas implantossuportadas.

O tratamento da síndrome da combinação objetiva estabelecer oclusão balanceada, minimizando a carga de oclusão na região anterior da maxila, devido aos antagonistas naturais inferiores. ${ }^{13,14}$

Os objetivos deste artigo são relatar, por meio de um caso clínico, as características clínicas presentes em um paciente desdentado total superior com a presença de dentes anteriores inferiores com contato dental anterior e apresentar uma proposta de tratamento reabilitador, restabelecendo a função fonética e a estética do paciente.

\section{Relato de caso}

Paciente, gênero masculino, 61 anos de idade, compareceu à Clínica de Reabilitação Oral do Curso de Odontologia da Universidade Federal do Ceará, Campus Sobral, CE, queixando-se de dificuldade mastigatória, uma vez que não fazia uso de próteses.

Ao exame clínico inicial, constatou-se que o paciente possuía dentes inferiores anteriores e ausência bilateral de dentes posteriores (classe I de
Kennedy). Apresentava também perda da dimensão vertical de oclusão e, na arcada inferior, dentes anteriores remanescentes extruídos. Observou-se perda óssea severa em região anterior da maxila, confirmada no exame radiográfico panorâmico. Analisando esses aspectos, foi possível obter o diagnóstico de síndrome da combinação ou síndrome de Kelly.

Após o diagnóstico e a confirmação das características clínicas pertinentes à síndrome da combinação, estabeleceu-se um plano de tratamento. $\mathrm{O}$ primeiro procedimento clínico foi a moldagem inicial das arcadas superior e inferior, utilizando-se alginato. Em seguida, foi realizado o recontorno incisal dos dentes anteriores inferiores em resina composta (Figura 1), com a utilização de mock-up para a reconstrução da face lingual dos dentes (Figura 2).

Para a confecção do mock-up, é necessária uma moldagem inicial bem realizada, a fim de se obter o modelo de estudo em que é realizado o enceramento diagnóstico, confeccionado a partir de um modelo de gesso encerado em laboratório, de acordo com as dimensões ideais de cada elemento dentário e de sua respectiva arcada, permitindo, assim, a reanatomização. Realizado o enceramento diagnóstico, faz-se um guia de transferência utilizando silicone pesado.

Subsequentemente, foi realizada a moldagem funcional das arcadas superior e inferior. Obtido o modelo de trabalho superior, confeccionou-se a base de prova em resina acrílica autopolimerizável; em seguida, instalou-se o plano de orientação, e determinaram-se suporte labial, corredor bucal, linha alta do sorriso, linha dos caninos e linha média. Após a obtenção do modelo de trabalho inferior, realizou-se o guia de transferência (Figura 3).

Determinada a trajetória de inserção no modelo de estudo, foi realizado o guia de transferência com uma placa de acrílico, selecionando 3 pontos, sendo dois posteriores e um anterior. $\mathrm{O}$ modelo foi isolado com vaselina, e inserida resina ativada quimicamente nos pontos escolhidos. Com auxílio do delineador, um prego foi fixado à superfície da placa de acrílico com godiva de baixa fusão. Posteriormente, foram executados o planejamento e a confecção da estrutura metálica inferior (Figura 4).

Figura 1 - Recontorno incisal dos dentes anteriores inferiores

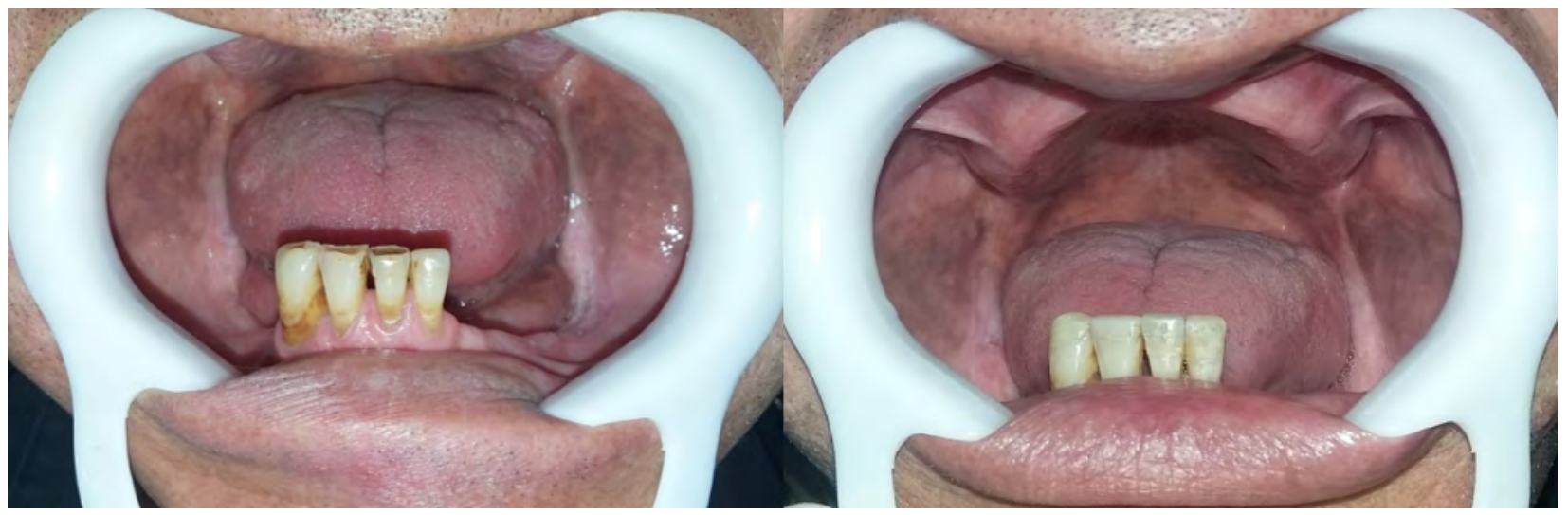

Fonte: autores. 


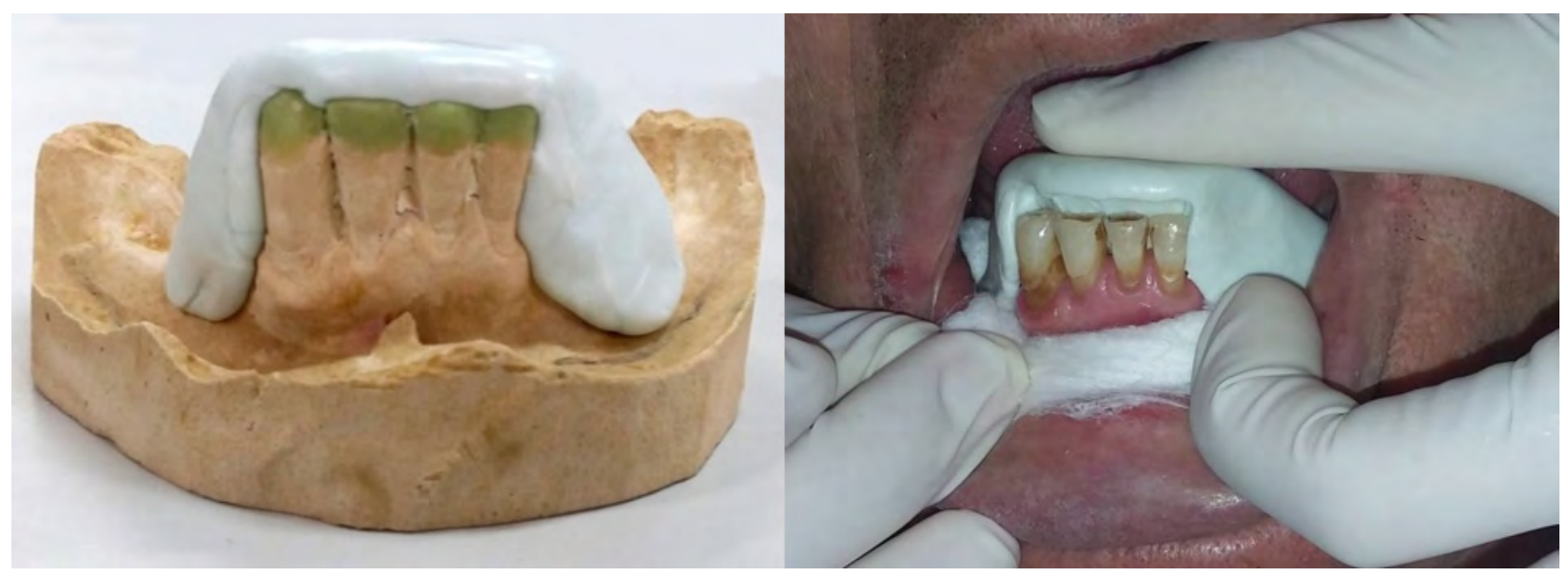

Fonte: autores.

Figura 3 - Guia de transferência em modelo

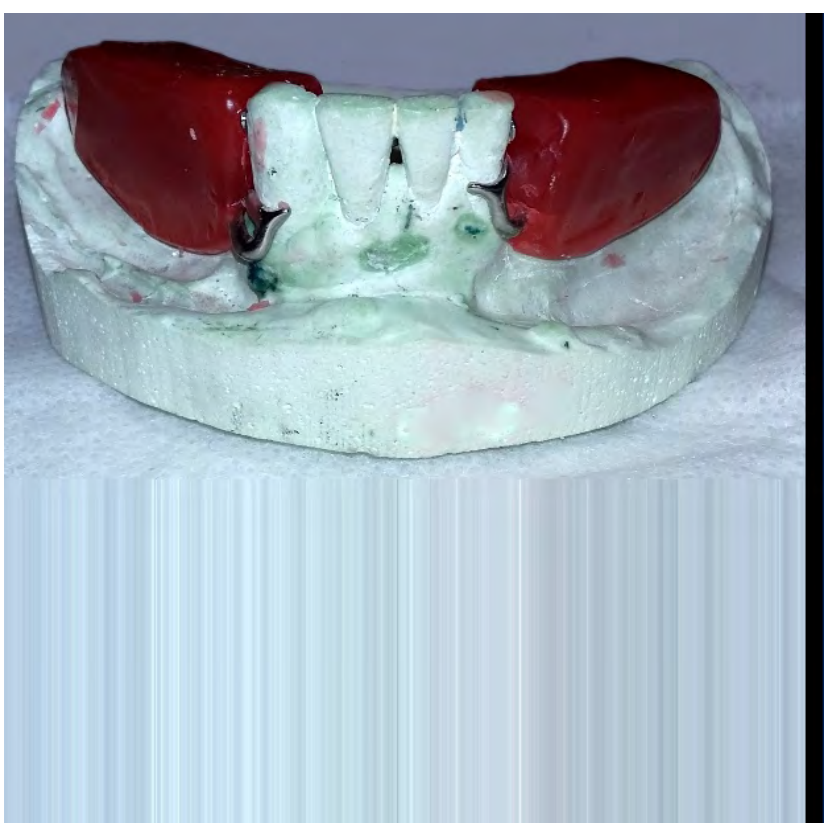

Fonte: autores.

Figura 4 - Estrutura metálica com cera de trabalho

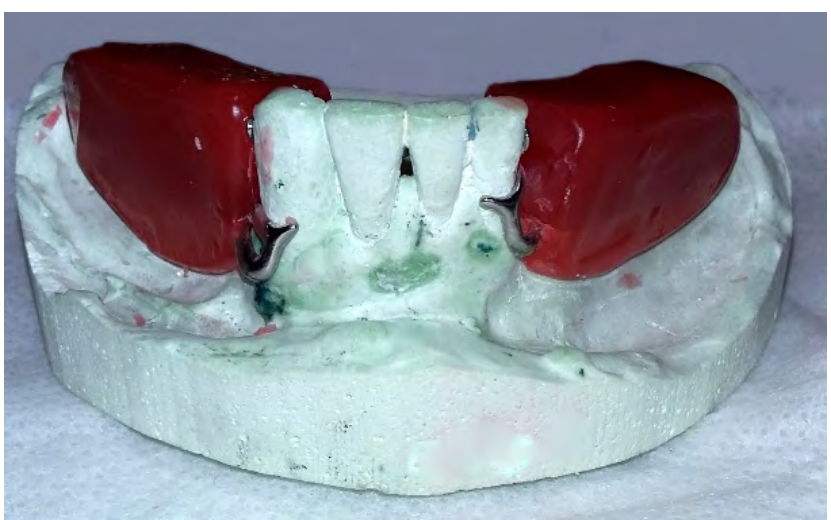

Fonte: autores.
Em um momento posterior, realizou-se a tomada do arco facial, e o articulador semiajustável foi enviado para o laboratório para a montagem dos dentes (Figura 5). Foram realizadas a prova dos dentes e a seleção da cor da gengiva, de acordo com a escala VIP Trilux. Em seguida, as peças foram enviadas para laboratório para a acrilização da prótese total superior e da prótese parcial removível inferior (Figuras 6 e 7). E, então, depois de acrilizadas, as próteses foram instaladas, objetivando restabelecer a função fonética e a estética do paciente (Figura 8).

Figura 5 - Montagem dos dentes em articulador semiajustável

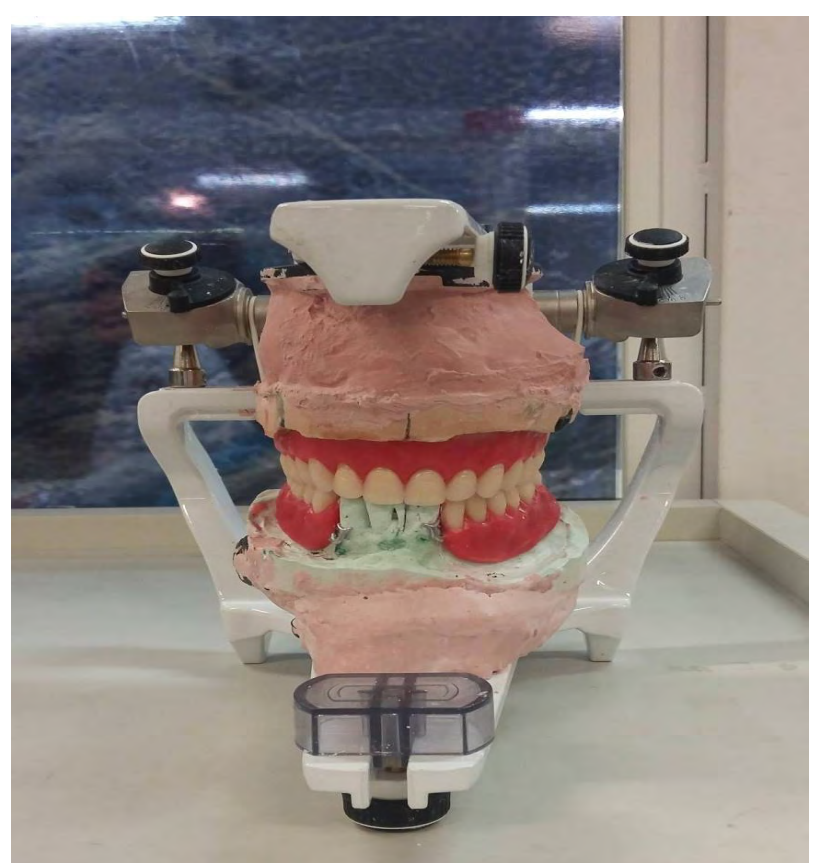

Fonte: autores. 
Figura 6 - Prótese total superior acrilizada

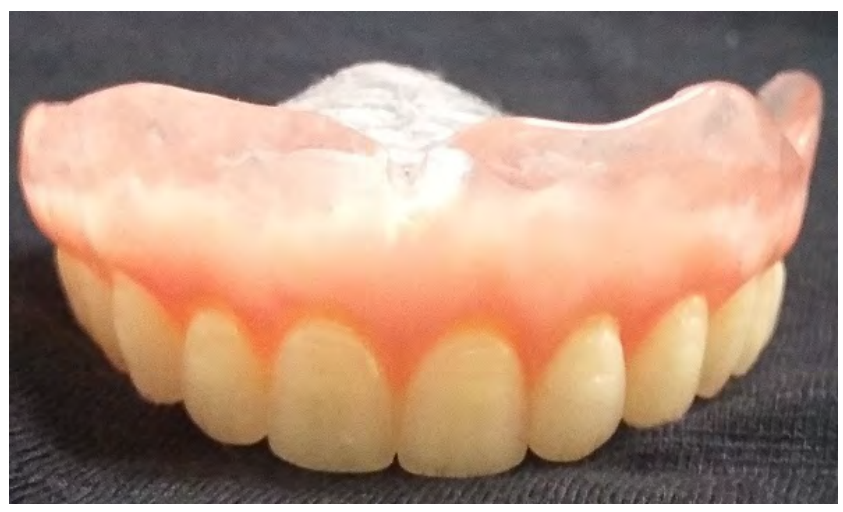

Fonte: autores.
Figura 7 - Prótese parcial removível acrilizada

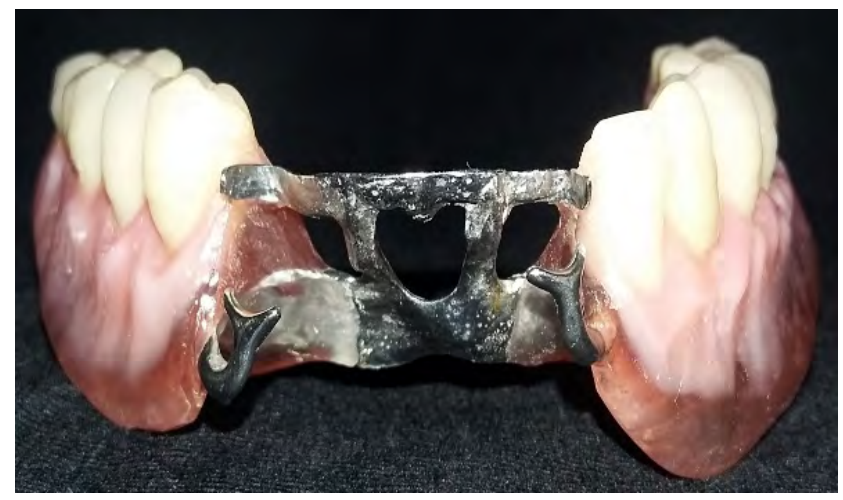

Fonte: autores.

Figura 8 - Aspectos inicial e final, intraoral e extraoral

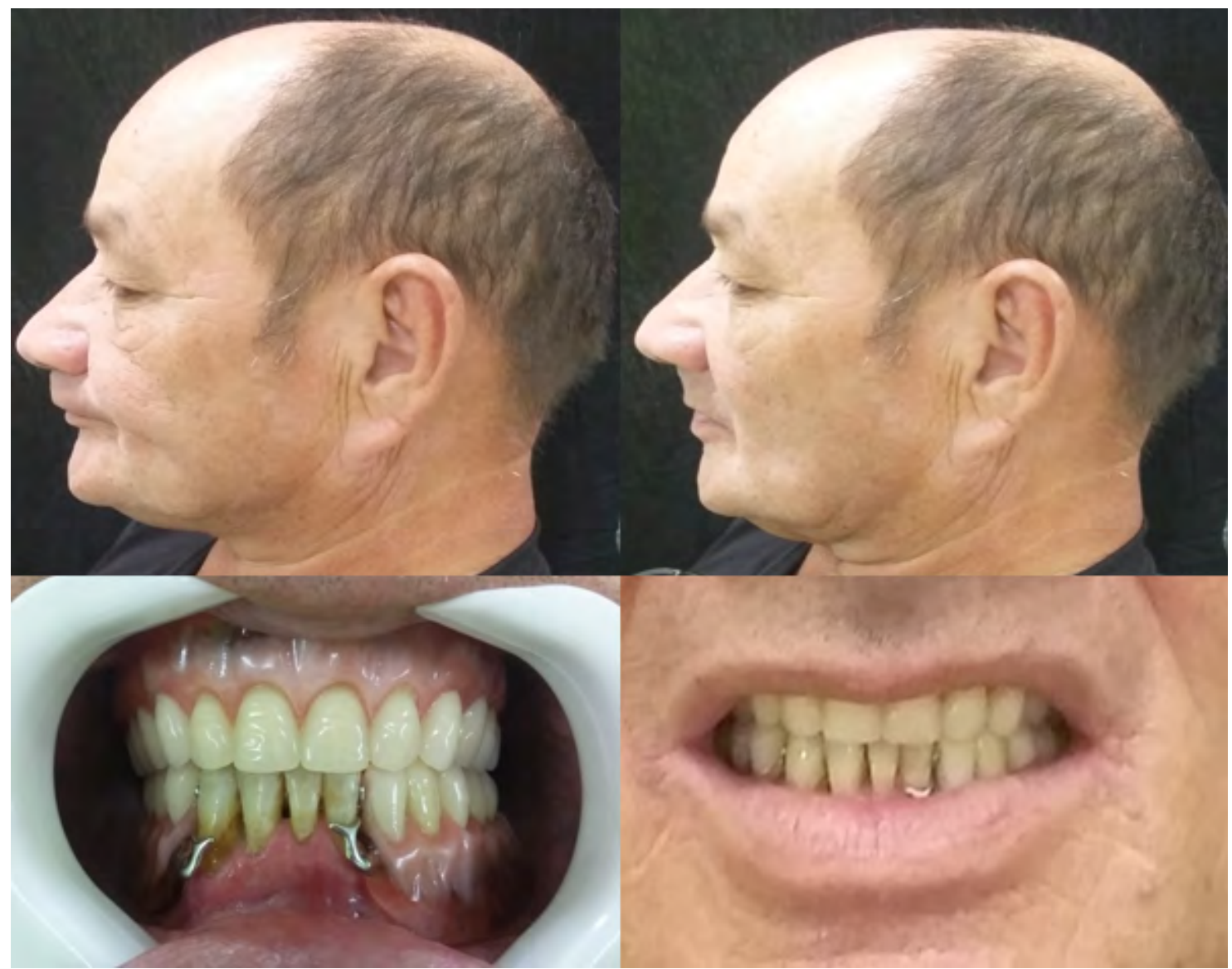

Fonte: autores.

\section{Discussão}

O diagnóstico prévio das manifestações clínicas relacionadas à síndrome da combinação, devidamente relatado no caso clínico, é crucial para o adequado tratamento reabilitador, de forma a restabelecer a função fonética e a estética do paciente. ${ }^{10,15}$ A literatura expõe sinais clínicos característicos, no entanto, ainda há um embate sobre quantos sinais simultaneamente são necessários para o diagnóstico da síndrome. ${ }^{7,8,11,15}$
Diversas são as abordagens de tratamento para pacientes com edentulismo na maxila e com presença de dentes anteriores na mandíbula. ${ }^{4,6,10} \mathrm{O}$ objetivo é o restabelecimento das funções do sistema estomatognático e do aspecto estético, assim, a escolha do tratamento depende da condição do quadro clínico do paciente, do status econômico e da opção do paciente pelo tipo de prótese, se fixa ou removível. $8,9,15$

Embora não seja a melhor escolha como tratamento para a síndrome da combinação, a prótese total superior em antagonista com a prótese parcial 
removível inferior é amplamente escolhida por pacientes, devido ao tempo clínico e ao baixo custo de confecção. ${ }^{10}$ Essa escolha pode promover aumento da força dos contatos anteriores e agravar o quadro do paciente, devido à reabsorção óssea do processo alveolar superior anterior, seguido da perda de retenção da prótese., ${ }^{4,7,9}$

Entre as maneira de reduzir a reabsorção óssea da pré-maxila anterior, estão o reembasamento e ajustes oclusais antes da reabilitação e acompanhamentos periódicos. ${ }^{11,12}$ Ainda assim, deve ser realizado um esquema oclusal em que haja uma oclusão mutuamente protegida e balanceada bilateral, livrando de pressão demasiada a região anterior da maxila. ${ }^{11-13}$

Salienta-se que, a partir dos implantes osseointegrados, é possível realizar o tratamento e prevenir possíveis agravos da condição clínica do paciente. Com o posicionamento de implantes na região posterior, evitam-se os extremos livres e as movimentações da prótese que aceleram a reabsorção óssea na região posterior da mandíbula. ${ }^{6,15}$

\section{Conclusão}

A reabsorção óssea alveolar edêntula é inevitável, quando se utilizam próteses mal adaptadas. O profissional deve diagnosticar, diante das características e dos sintomas da síndrome da combinação, o caso e realizar um tratamento reabilitador, de acordo com cada particularidade do paciente, devolvendo harmonia ao sistema estomatognático, função fonética e estética.

\section{Abstract}

Objective: treatment of patients with reactivity with respiratory problems. Identified by Kelly in 1972, a combination syndrome and an intraoral manifestation found in fully edentulous patients in the upper arch as opposed to a mandibular prosthesis of bilateral distal extension. Case report: the present study reviews and discusses, through a clinical case, as clinical features present in a patient with manifestations of the combination syndrome, in addition, to present a proposal of rehabilitative treatment, restoring function, phonetics and esthetics to the patient. Final considerations: diagnosing a syndrome and establishing a treatment plan appropriate to the needs of the patient and creating clinical conditions for the reestablishment of health are essential to obtain the result and thus impeding the advancement of the clinical picture.

Keywords: Diagnostic. Kelly syndrome. Combination syndrome.

\section{Referências}

1. Palmqvist S, Carlsson GE. The combination syndrome: a literature review. The J Prost Dentistry 2003; 90(3):270-5.

2. Schmitt SM. Combination syndrome: a treatment approach. The J Prost Dentistry 1985; 54(5):664-71.

3. Kelly E. Changes caused by a mandibular removable partial denture opposing a maxillary complete denture. The J Prost Dentistry 1972; 27(2):140-50.

4. Saunders TR, Gillis RE, Desjardins RP. The maxillary complete denture opposing the mandibular bilateral distalextension partial denture: treatment considerations. The $J$ Prost Dentistry 1979; 41(2):124-8.

5. Tolstunov L. Combination syndrome: classification and case report. J Oral Implantology 2007; 33(3):139-51.

6. Tolstunov L. Management of biomechanical complication of implant-supported restoration of a patient with combination syndrome: a case report. J Oral and Maxillofac Surg 2009; 67(1):178-88

7. Shen K, Gongloff RK. Prevalence of the 'combination syndrome' among denture patients. The J Prost Dentistry 1989; 62(6):642-4.

8. Goyatá FR, Gonçalves PAM, Rodrigues CRT, Souza MCA. Reembasamento em prótese total: relato de caso clínico. Dent Sci 2009; 3(9):39-46.

9. Cunha LDAP, Rocha EP, Pellizzer EP. Prevalência da Síndrome de Kelly em usuários de prótese parcial removível. Rev Gaúcha Odontol 2007; 55(4):325-32.

10. Salvador MCG, Valle ALD, Ribeiro MCM, Pereira JR. Assessment of the prevalence index on signs of combination syndrome in patients treated at Bauru School of Dentistry, University of Sao Paulo. J Applied Oral Science 2007; 15(1):9-13.

11. Cabianca M. Combination syndrome: treatment with dental implants. Implant Dentistry 2003; 12(4):300-5.

12. Shanthi PK, Reddi NRM, Raja RN, Sunil M. Prosthodontic rehabilitation of a patient with combination syndrome. Indian Journal of Dental Advancements 2015; 7(1):58-62.

13. Costa MM, Cardoso GM, Júnior LHF, Resende CCD, Tavares LN, Rocha FS, et al. Síndrome da combinação: diagnóstico, prevenção e considerações sobre o tratamento. Rev Odontol Brasil Central 2016; 25(72):59-64.

14. Zanetti GR, Castro GCD, Borges-Filho FF, Peçanha MM, Zanetti LSDS. Guia cirúrgico modifcado para tuberoplastia em pacientes com Síndrome da Combinação. Rev Odontol UNESP 2010; 39(2):131-5.

15. Resende CMBMD, Ribeiro JAM, Dias KDC, Carreiro ADFP, Rego MPPD, Queiroz, JWND, et al. Signs of Combination Syndrome and removable partial denture wearing. Rev de Odontologia da UNESP 2014; 43(6):390-5.

\section{Endereço para correspondência:}

Manassés Tercio Vieira Grangeiro

Av. Eng. Francisco José Longo, 777, São Dimas

12245-000, São José dos Campos, SP, Brasil

Telefone: (88) 99906-0612

E-mails: manasses.grangeiro@ict.uneso.br terciomanasses@gmail.com 